\title{
An Automated Performance-Aware Approach to Reliability Transformations
}

\author{
Jacob Lidman ${ }^{1}$, Sally A. McKee ${ }^{1}$, Daniel J. Quinlan ${ }^{2}$, and Chunhua Liao ${ }^{2}$ \\ 1 Department of Computer Science and Engineering \\ Chalmers University of Technology \\ Gothenburg, Sweden \\ \{lidman, mckee\}@chalmers \\ 2 Lawrence Livermore National Laboratory \\ Livermore, CA, USA \\ \{dquinlan,liao6\}@llnl.gov
}

\begin{abstract}
Soft errors are expected to increase as feature sizes shrink and the number of cores increases. Redundant execution can be used to cope with such errors. This paper deals with the problem of automatically finding the number of redundant executions needed to achieve a preset reliability threshold. Our method uses geometric programming to calculate the minimal reliability for each instruction while still ensuring that the reliability of the program satisfies a given threshold. We use this to approximate an upper bound on the number of redundant instructions. Using this, we perform a limit study to find the implications of different redundant execution schemes. In particular we notice that the overhead of higher redundancy has serious implications to reliability. We therefore create a scheme where we only perform more executions if needed. Applying the results from our optimization improves reliability by up to $58.25 \%$. We show that it is possible to achieve up to $8 \%$ better performance than Triple Modular Redundancy (TMR). We also show cases where our approach is insufficient.
\end{abstract}

Keywords: High Performance Computing, Fault Tolerance, N-Modular Redundancy, Reliability Optimization.

\section{Introduction}

Technology trends like shrinking feature sizes and increasing numbers of processor cores on chip make transient faults in hardware increasingly common. These transient faults manifest themselves as bit-flips, and they can originate from external sources (e.g., radiation events) or internal sources (e.g., voltage drops, power supply noise, or leakage). Such faults are termed soft errors because they cause no permanent device damage.

As hardware increases in complexity, so does the software that runs on it. Increased complexity results in increased error vulnerability at all levels of the software stack. These problems affect all segments of computing, but they are of particular concern for High Performance Computing (HPC) platforms that

L. Lopes et al. (Eds.): Euro-Par 2014 Workshops, Part I, LNCS 8805, pp. 523534 2014.

(C) Springer International Publishing Switzerland 2014 
must continue to perform correctly in the presence of such faults. Architects have long proposed hardware enhancements [1] to improve fault tolerance, but implementing dedicated hardware solutions requires design and verification effort, consumes chip real estate, and increases hardware complexity: at some point the proposed solution itself becomes part of the problem. Furthermore, large-scale supercomputers have historically been built from commodity parts not designed for use at such scales; as such, they lack dedicated hardware support, and adding it "after the fact" is difficult and impractical, if not impossible.

Scalable software solutions could become attractive alternatives, but software approaches can incur high performance overheads. As with dedicated faulttolerant hardware solutions, the fault-tolerant software itself can potentially introduce new errors, since it adds to the (growing) complexity of the application software. To balance these tradeoffs, we study the problem of automatically introducing fault tolerance in software to correct for hardware-induced faults.

Software-implemented hardware fault tolerance (SIHFT) typically relies on adding some form of redundancy. This could entail re-executing instructions when faults are detected (temporal redundancy) or executing multiple independent instructions and adjudicating on their results (spatial redundancy) [15]. The former approach is typically called checkpointing or replay, and HPC systems have traditionally employed it at multiple levels (e.g., in both the OS and the application). Engelmann et. al [2] argue that as the number of nodes increases, system availability will decrease due to single node failures. Increasing the number of nodes also increases the time it takes to save and restore the state when faults occur. The latter approach is termed $N$-modular redundancy, or NMR (where $N$ signifies the number of independent instructions). In both cases, the amount of work a processing element needs to perform increases, which increases the opportunities for incurring soft errors. An understanding of the tradeoffs between reliability and performance is necessary to make efficient use of hardware resources while delivering a desired level of resilience.

To understand the motivation for automatically deducing $N$, consider a program in which an instruction $I_{X}$ consumes the results produced by an instruction $I_{Y}$. The probability that $I_{X}$ produces the correct result can be increased by either hardening $I_{X}$ or $I_{Y}$. In particular, if executing $I_{X}$ comes at a lower performance cost than executing $I_{Y}$, we may prefer to harden the former. In extending to even more instructions, we need to consider how results propagate: producers with more consumers need more protection. The typical approach to applying NMR using a fixed number of redundant executions for all instructions neglects this issue. If $I_{X}$ and $I_{Y}$ are redundantly executed different numbers of times, then we also need a means by which to decide how to propagate results when there are fewer consumers than producers (or vice versa). Here we develop an automated approach to determining an appropriate level of redundancy for each instruction and leave the second problem of deciding the interconnection between producers and consumers for future work. We therefore combine producer results before consumer instructions need them. 


\section{Related Work}

Checkpointing has long been the standard approach to hardening HPC applications. Application and system state are periodically saved at dedicated nodes. In the event of a crash, the application is rolled back to a committed state. Lu et. al. 7] highlight the need to keep multiple checkpoint versions to deal with latent errors: this lets them restart in a stable state from before an error was generated, even if the time between occurrence and detection exceeds a single checkpoint interval. The overheads of checkpoint/restart are expected to cause system utilization to decrease rapidly as systems grow to exascale 3]. Researchers are thus investigating a limited amount of modular redundancy, as it allows some soft errors to be handled by a local node cluster, rather than invoking a global restart 345]. These approaches use a static number of redundant executions.

Minimizing the performance impact of resilient code is naturally important to the HPC community. Shamsunder et. al. [6] consider the problem of minimizing the number of assertion checks in a multiprocessor environment. They use a CFG-like graph model to represent computation and find an efficient algorithm for the case in which the number of faults is fixed. In contrast, our approach uses a probabilistic description of whether an operation suffers a fault. Misailovic et. al. 8] present an algorithm for replacing operations with less reliable versions while minimizing power consumption and maintaining a preset reliability. Their approach uses a formulation of the optimization problem similar to ours, but they use Integer Linear Programming (ILP) to solve it.

\section{Approach}

We want to generate code delivering a specified level of reliability while minimizing the performance costs of redundant execution. Our approach is based on the duality between a program's data flow and the probabilistic flow, or how the probability of a state's being correct increases/decreases as it is altered by operations. All data-flow operations increase execution time. Similarly, in the probabilistic interpretation, all non-ideal operations degrade the reliability of producing a correct output. We restrict ourselves to cases where reliability and performance are linearly related. By viewing the operations as constraints on the probabilistic flow, we can find a solution that satisfies a reliability threshold while minimizing performance overheads.

Let a program be represented by a control-flow graph (CFG) $G=\langle V, E\rangle$. Each block $v \in V$ is a sequence of binary $\left(r_{D}=I_{o p}\left(r_{S}, r_{T}\right)\right)$ or unary $\left(r_{D}=I_{o p}\left(r_{S}\right)\right)$ operations over a finite state-space of non-overlapping symbols from a set $R$. Informally, the semantics of a unary (binary) data-flow operation is that $r_{D}$ is the destination symbol of an operation $I_{o p}$ on symbols $r_{S}$ (and $r_{T}$ ). We use functions $f^{V}$ to map a symbol $r_{x}$ to a variable that symbolically represents the probability that $r_{x}$ is correct.

Associated with all unary (binary) data-flow operations is a probability $P_{I_{o p}}$ that $I_{o p}$ computes correctly given correct operands. Although one would generally expect this probability to change with the operand values, it is common to 
assume that the failure of the operation and its operands are independent 10 . We denote the probability of successful execution prior to optimization as $P_{I_{o p}}^{\text {init }}$, which we refer to as the initial execution probability of $I_{o p}$. Similarly, we refer to the value of $P_{I_{o p}}$ following optimization as the optimal execution probability of $I_{o p}$. The probability that a binary operation $I_{o p}$ produces the correct result (for some $\left.f^{V}\right)$ is then given by $P_{I_{o p}} f^{V}\left(r_{S}\right) f^{V}\left(r_{T}\right)$.

\subsection{Optimization Problem}

Algorithm 1 translates a program into a set of constraints that reflect the probability that the data flow is correct. The function new $\operatorname{Var}()$ returns a fresh variable, and Constraint $\left(o p, v_{I d}, f^{V}, r_{D}, S, C\right)$ adds a constraint 11 of the form $1=v_{I d}^{-1} P_{I_{o p}} \prod_{s \in S} f^{V}(s)$ to $C$ and maps $r_{D}$ to $v_{I d}$ in $f^{V}$. The $P_{I_{o p}}$ variables differ from the $v_{x}$ variables in that we associate a weight $w_{o p}$ with the former. The goal of the optimization process is to minimize $\sum_{i} w_{i} P_{I_{i}}$ subject to the constraints generated by the algorithm, namely that each variable $\in[0,1]$, and that the reliability of important symbols reach a predefined level $\hat{P}$. Such an optimization problem is called a geometric programming problem. The resulting (optimal) $P_{I_{i}}$ is later used to find an appropriate $N$ for each instruction.

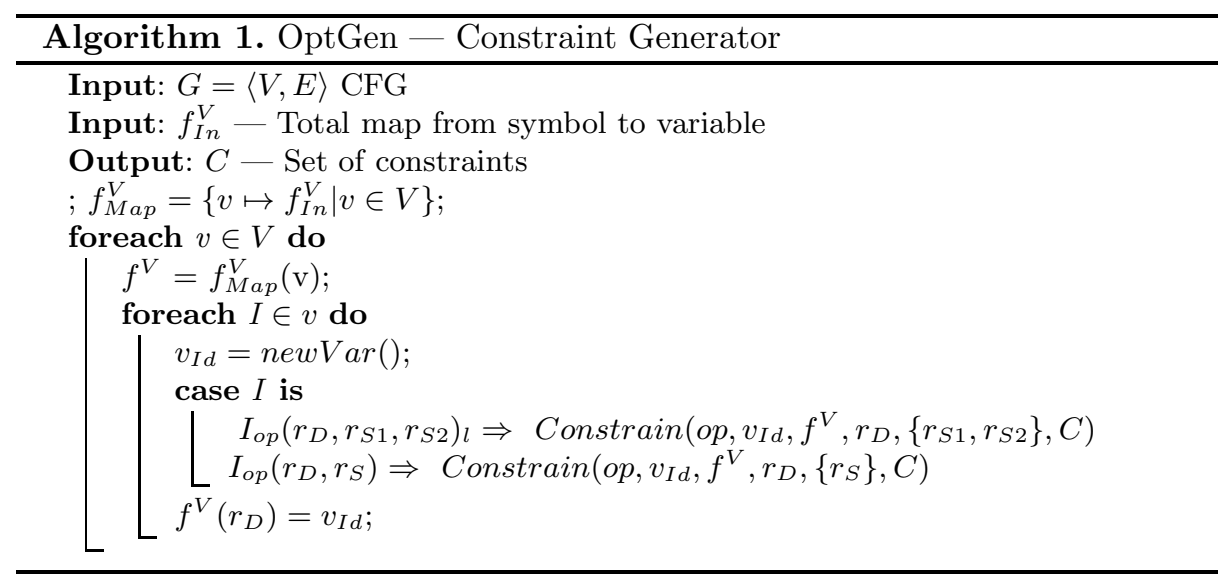

Let $c>0, x_{i} \in \mathbb{R}^{+}$and $a_{i} \in \mathbb{R}$. Then $g_{k}(\boldsymbol{x})=c \prod_{i} x_{i}^{a_{i}}$ is a mononomial function. Similarly $f_{j}(\boldsymbol{x})=\sum_{i} g_{i}(\boldsymbol{x})$ is a posynomial function. A geometric programming (GP) problem in standard form is given by:

$$
\min f_{0}(x) \text { s.t. }\left(\forall j \in[1, m]: f_{j}(x) \leq 1\right) \wedge\left(\forall k \in[1, p]: g_{k}(x)=1\right)
$$

GP problems can be transformed into a convex equivalent form by taking the logarithm of all posynomials. The resulting functions are called log-sum-exp

${ }^{1}$ These constraints impose restrictions on the probabilistic flow of an instruction with destination symbol $r_{D}$ and source symbols $s \in S$. 


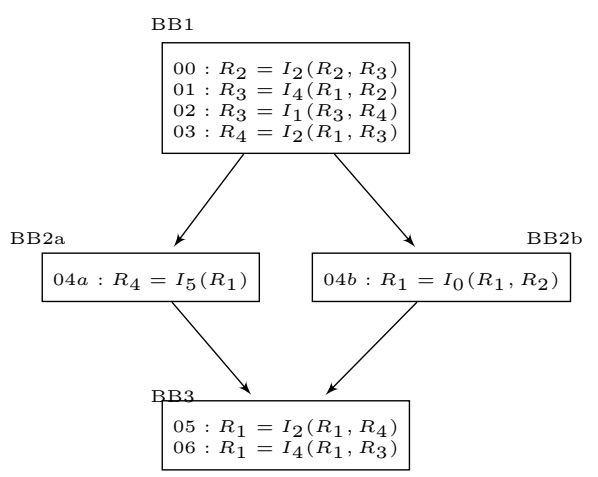

$$
\begin{aligned}
& \min w^{T} P_{O p} \\
& \text { s.t. } 1=v_{00}^{-1} P_{o p(0)}{ }^{v} R_{2}{ }^{v} R_{3} \\
& 1=v_{01}^{-1} P_{o p(1)} v_{R_{1}} v_{00} \\
& 1=v_{02}^{-1} P_{O P(2)} v_{01} v_{R 4} \\
& 1=v_{03}^{-1} P_{o p(3)} v_{R_{1}} v_{02} \\
& 1 \geq v_{00}^{-1} \hat{P} \\
& 1 \geq v_{02}^{-1} \hat{P} \\
& 1 \geq v_{03}^{-1} \hat{P} \\
& 1=v_{04 a}^{-1} P_{o p(4 a)}{ }^{v} R_{1} \\
& 1 \geq v_{04 a}^{-1} \hat{P} \\
& 1=v_{04 b}^{-1} P_{o p(4 b)} v_{R_{1}} v_{R_{2}} \\
& 1 \geq v_{04 b}^{-1} \hat{P} \\
& 1=v_{05}^{-1} P_{o p(5)} v_{R_{1}} v_{R_{4}} \\
& 1=v_{06}^{-1} P_{o p(6)} v_{05} v_{R_{3}} \\
& 1 \geq v_{06}^{-1} \hat{P} \\
& v_{*} \leq 1 \\
& P_{o p(*)} \leq 1 \\
& \forall i: 1 \geq P_{o p(i)}^{-1} P_{o p(i)}^{i n i t}
\end{aligned}
$$

Fig. 1. Example CFG and Corresponding GP Optimization Problem

functions (i.e., $\left.y=\log \left(\sum_{i} e_{i}^{x}\right)\right)$. Off-the-shelf solvers [9] can find the minimum of a GP problem in convex form efficiently even for large numbers of variables. Note that the convex form of a GP problem of only monomial functions, as in our case, is a linear programming problem.

As an example, consider the CFG in Figure 3.1, Variables $\left\{v_{R_{0}}, v_{R_{1}}, v_{R_{2}}, v_{R_{3}}\right.$, $\left.v_{R_{4}}\right\}$ denote the probability that symbols $R_{0}, R_{1}, R_{2}, R_{3}$ and $R_{4}$, respectively, are correct at the entry of a block. The corresponding GP problem is given to the right. We add constraints (for symbols that have been updated in the block) to enforce the requirement that the probability of correctness reaches at least $\hat{P}$. The reliability of an NMR system with majority voting as the adjudication mechanism and a component reliability of $p$ is given by $\sum_{j=n+1}^{N}\left(\begin{array}{c}N \\ j\end{array}\right) p^{j}(1-p)^{N-j}$ (where $N=2 n+1)$. Using this we calculate the necessary number of redundant instructions (with initial and optimal execution probabilities $p_{i}$ and $p_{o}$, respectively) by finding the lowest $N$ such that $p_{o} \leq \sum_{j=n+1}^{N}\left(\begin{array}{c}N \\ j\end{array}\right) p_{i}^{j}\left(1-p_{i}\right)^{N-j}$.

\subsection{1.mn Voter}

The number of redundant executions our approach recommends can be quite high if $\hat{P}$ is high and/or $P_{o p}$ is low. As these numbers may represent pessimistic estimates of the number of situations in which faults occur, we introduce a voter system that adds redundant executions only as needed.

The voting system consists of $n$ stages (for some $n \in \mathbb{N}-\{0\}$ ). Each stage includes a sequence of redundant executions followed by an assertion that tries 
to establish whether more executions are needed. Listing 1.1 shows pseudocode for the voting system (here $x=f(\ldots)$ is the computation we want to protect). In the first stage, we execute $m+1$ versions (from some even $m>0$ ). We compare these $m+1$ results using a parity function that is zero iff an even number of operands are ones. If zero, we vote on the result. Otherwise, we replace the result of the first version with the vote of all results in the first stage and perform $m$ more executions in a second stage. This repeats for a maximum of $n$ stages. We refer to this voter system as the $1 . m n$ voter.

Listing 1.1. 1.mn Voter System

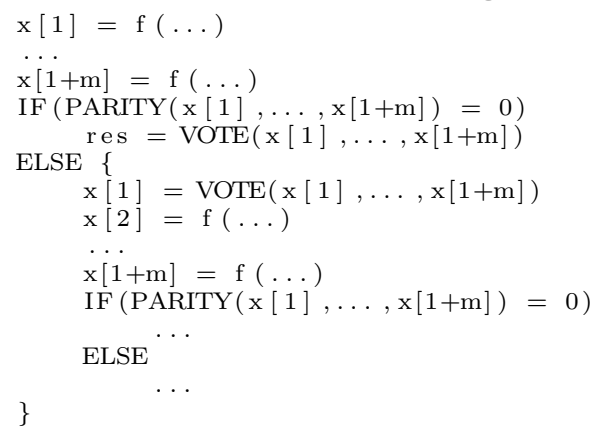

\section{Evaluation}

To compare our approach to assigning a fixed number of redundant executions, we use the matrix kernels listed in Table1. We choose these kernels to show benefits and weaknesses of our approach rather than based on application domain.

Table 1. Evaluation Kernels

\begin{tabular}{|c|c|c|}
\hline Name & Description & Matrix Size \\
\hline Approx-log & Approximates the logarithm of fix-point numbers in the range $[0,1]$ & $5 \times 5$ \\
\hline Determinant & Computes the determinant by Laplace expansion recursively & $5 \times 5$ \\
\hline Gaussian & Implements convolution with a $9 \times 9$ Gaussian smoothing kernel & $10 \times 10$ \\
\hline Bubblesort & Implements Median filtering using $3 \times 3$ bubblesort sorting network & $10 \times 10$ \\
\hline Mincomp & Implements Median filtering using $3 \times 3$ minimum comparison sorting network & $10 \times 10$ \\
\hline Mintime & Implements Median filtering using $3 \times 3$ minimum time sorting network & $10 \times 10$ \\
\hline
\end{tabular}

\subsection{Experimental Setup}

In our experiments we use the gem5 simulator [14 to model an in-order 32-bit MIPS processor. We modify the simulator to include a pseudo-random number generator for flipping bits in the inputs of the integer ALU in the execution stage. Faults are only injected into instructions that belong to the application. Instructions that do not involve ALU units (e.g., memory loads/stores or moving data between registers) are considered ideal, since effective hardware fault-tolerance methods exist for these cases (e.g., redundant buses or error-correcting codes). 
The random variable controlling which ALU unit to zap is exponentially distributed with the unit's normalized area and a constant $\lambda_{k}$ (i.e., $\left.1-e^{-\lambda_{k} \frac{A r e a u n i t}{A r e a} A L U}\right)$. The flip probability of each unit is given in Table 3. The initial successful execution probabilities, $P_{I_{o p}}^{\text {init }}$, similarly use these probabilities. We vary the constant $\lambda_{k}$ across experiments to conduct a limit study. Which input bit to flip (of an ALU unit) is uniformly distributed. To obtain area numbers we synthesize an in-house MIPS implementation with the Synopsys Design Compiler using a commercial $65 \mathrm{~nm}$ low-power process technology. This implementation models a five-stage MIPS R3000-like processor.

Accessing pages that have not been allocated makes the OS terminate the process. This is a common case when injecting faults [15. To avoid such terminations, we change the gem 5 virtual memory system so that pages are allocated before we read/write memory. Reading from an invalid memory address then returns a non-deterministic value. This concept has been called failure-oblivous computing [16. With these changes, an invalid memory access can be handled with error-correction logic, as opposed to terminating the execution.

\subsection{Methodology}

We compile our kernels with GCC 4.0.12 and optimization level 0 (-O0) to include DWARF debugging information $(-\mathrm{g})$. We analyze the binaries with ROSE [1] to produce a GP problem, as described in Section 3 We require that the probability of correctness of each 32-bit register assigned in the block reaches at least $\hat{P}=0.99$. For the sake of demonstration, we use unit weights for all ALU instructions except $\operatorname{div} / \mathrm{mul}$, for which we use 10 (since they are typically about an order of magnitude higher in performance cost). We use CVX 2.0 beta [1213] in MATLAB R2012 to solve the GP problem and return the resulting optimal execution probabilities to ROSE. For each pair of initial and optimal execution probabilities we approximate an upper bound on the number of redundant executions, $N$, for each instruction. Since ROSE::FTTransform [15] operates on source code, we use the DWARF sourceline-instruction address mapping to transfer this number back to the source level. Since source lines map to multiple instructions, we use the maximum $N$ of all instructions mapping to a particular source line. We use a $1.2 N_{i}$ voter for each source line, where $N_{i}$ versions were recommended if $N_{i}>1$. For $N_{i}=1$ we do not apply any transformation. Table 2 shows the values of $N$ for each kernel.

For each kernel we use ROSE::FTTransform to produce two sets of versions. The first set contains the results of applying each kernel to a transformer in which we use $3 \mathrm{MR}, 5 \mathrm{MR}$, and $7 \mathrm{MR}$ versions with majority voting. The second set uses a $1.2 n$ voter, where $n$ is set to $\{2,3,4\}$ to produce versions called 1.2$2,1.2-3$, and 1.2-4. These two sets and the set of versions produced by our

\footnotetext{
${ }^{2}$ This particular toolchain is supported by gem5's MIPS simulator in system-call emulation mode.

${ }^{3}$ We arbitrarily choose this value for the purposes of our limit study.
} 
Table 2. Distributions of $N$ for All Source Lines of Each Kernel

\begin{tabular}{|l|l|l|l|l|l|l|}
\hline & \multicolumn{4}{|c|}{1.0} & \multicolumn{1}{c|}{0.1} & \multicolumn{1}{c|}{0.01} \\
\hline & \multicolumn{3}{|c|}{$\lambda_{k}$} \\
\hline Name & $\tilde{N}$ & N Values & $\tilde{N}$ & N Values & $\tilde{N}$ & N Values \\
\hline Approx-Log & 3 & $\{1,3,7\}$ & 3 & $\{1,3\}$ & 3 & $\{1,3\}$ \\
\hline Determinant & 3 & $\{1,3,7,9,23\}$ & 3 & $\{1,3,5,9\}$ & 3 & $\{1,3\}$ \\
\hline Gaussian & 31 & $\{1,3,19,23,27,31\}$ & 13 & $\{1,3,9,11,13\}$ & 9 & $\{1,3,5,7,9\}$ \\
\hline Bubblesort & 3 & $\{1,3,5\}$ & 3 & $\{1,3\}$ & 3 & $\{1,3\}$ \\
\hline Mincomp & 7 & $\{1,3,7,9\}$ & 5 & $\{1,3,5,7\}$ & 3 & $\{1,3,5\}$ \\
\hline Mintime & 7 & $\{1,3,7,9,11\}$ & 5 & $\{1,3,5,7\}$ & 3 & $\{1,3,5\}$ \\
\hline
\end{tabular}

Table 3. Flip probabilities for ALU units

\begin{tabular}{|l|r|}
\hline ALU Unit & P(flip) \\
\hline Add/Sub & $1-0.998766$ \\
\hline Mul/Div & $1-0.953567$ \\
\hline Comparator & $1-0.999705$ \\
\hline And & $1-0.999799$ \\
\hline Or & $1-0.999778$ \\
\hline Xor & $1-0.999685$ \\
\hline Nor & $1-0.999852$ \\
\hline Shift & $1-0.998343$ \\
\hline
\end{tabular}

optimizer (called OPT) and the original kernel (called Orig), collectively make up the kernel versions considered in this work.

For each kernel version we then perform an experiment consisting of 2000 runs with $\lambda_{k} \in\{1,0.1,0.01\}$. We choose the values of $\lambda_{k}$ s.t. reliability results of the evaluations of the original kernel versions map to the range $[0 \%, 100 \%]$. Each run is classified as correct or incorrect. The incorrect class includes runs that time out (meaning the kernel did not complete within one minute4), encounter miscellaneous errors (e.g., control-flow errors or invalid syscall requests), and terminate with incorrect results (i.e., silent data corruptions). For each experiment we use the percentage of runs that are classified as correct to measure reliability. Similarly, we compute the median of the number of executed CPU cycles (as reported by gem5) of all correct runs, which we use to represent performance.

Table 4. Reliability Results for Each Kernel Version at Each $\lambda_{k}(1,0.1,0.01)$

\begin{tabular}{|c|c|c|c|c|c|c|c|c|c|}
\hline /ersion & \multicolumn{9}{|c|}{ Kernel } \\
\hline & \multicolumn{3}{|c|}{ Approx-Log } & \multicolumn{3}{|c|}{ Determinant } & \multicolumn{3}{|c|}{ Gaussian } \\
\hline & $k_{k}=1.0$ & $\lambda_{k}=0.1$ & $\lambda_{k}=0.01$ & $\lambda_{k}=1.0$ & $\lambda_{k}=0.1$ & $\lambda_{k}=0.01$ & $\lambda_{k}=1.0$ & $\lambda_{k}=0.1$ & $\lambda_{k}=0.01$ \\
\hline rig & $0.00 \%$ & $29.85 \%$ & $80.60 \%$ & $0.20 \%$ & $39.50 \%$ & $84.40 \%$ & $0.00 \%$ & $0.60 \%$ & $44.65 \%$ \\
\hline & & $\%$ & & $0 \%$ & 56.4 & & & & \\
\hline पR & & $34.70^{\circ}$ & & $5 \%$ & $49.30 \%$ & 82. & $0.00 \%$ & $36.15 \%$ & $0 \%$ \\
\hline$\overline{\mathrm{MR}}$ & $0.00 \%$ & $1.50 \%$ & $21.80 \%$ & $0.05 \%$ & $23.10 \%$ & $61.90 \%$ & $0.00 \%$ & $1.60 \%$ & $20.15 \%$ \\
\hline PT & $5.30 \%$ & $69.90 \%$ & $91.05 \%$ & $1.10 \%$ & $59.00 \%$ & $90.75 \%$ & $0.00 \%$ & $0.00 \%$ & $0.00 \%$ \\
\hline $2-2$ & $3.40 \%$ & $66.90 \%$ & $90.25 \%$ & $0.95 \%$ & $55.90 \%$ & $88.70 \%$ & $4.40 \%$ & $73.10 \%$ & $91.95 \%$ \\
\hline $2-3$ & $3.50 \%$ & $67.40 \%$ & $90.40 \%$ & $1.20 \%$ & $56.90 \%$ & $89.25 \%$ & $3.55 \%$ & $74.95 \%$ & $92.15 \%$ \\
\hline $1.2-4$ & $3.70 \%$ & $67.55 \%$ & $90.50 \%$ & $0.90 \%$ & $59.20 \%$ & $90.85 \%$ & $4.90 \%$ & $72.85 \%$ & $91.30 \%$ \\
\hline Version & \multicolumn{9}{|c|}{ Kernel } \\
\hline & \multicolumn{3}{|c|}{ Mincomp } & \multicolumn{3}{|c|}{ Mintime } & \multicolumn{3}{|c|}{ Bubblesort } \\
\hline & ${ }_{k}=1.0$ & $\lambda_{k}=0.1$ & $\lambda_{k}=0.01$ & $\lambda_{k}=1.0$ & $\lambda_{k}=0.1$ & $\lambda_{k}=0.01$ & $\lambda_{k}=1.0$ & $\lambda_{k}=0.1$ & $\lambda_{k}=0.01$ \\
\hline rig & $0.00 \%$ & $21.65 \%$ & $76.30 \%$ & $0.00 \%$ & $25.25 \%$ & $76.65 \%$ & $0.00 \%$ & $19.60 \%$ & $73.20 \%$ \\
\hline VR & $9.95 \%$ & $79.95 \%$ & 94.30 & $11.45 \%$ & $79.95 \%$ & $94.40 \%$ & $8.65 \%$ & $77.45 \%$ & $93.15 \%$ \\
\hline$\overline{\mathrm{MR}}$ & $1.45 \%$ & $58.75 \%$ & 83.60 & $1.65 \%$ & $58.25 \%$ & $82.85 \%$ & $0.45 \%$ & $48.65 \%$ & $77.45 \%$ \\
\hline $7 \mathrm{MR}$ & $0.00 \%$ & $18.45 \%$ & $51.10 \%$ & $0.00 \%$ & $7.65 \%$ & $38.75 \%$ & $0.00 \%$ & $8.30 \%$ & $36.80 \%$ \\
\hline OPT & $12.95 \%$ & $79.90 \%$ & $94.35 \%$ & $12.30 \%$ & $79.45 \%$ & $95.10 \%$ & $9.90 \%$ & $76.75 \%$ & $92.60 \%$ \\
\hline $1.2-2$ & $11.60 \%$ & $81.50 \%$ & $95.25 \%$ & $11.45 \%$ & $79.95 \%$ & $95.20 \%$ & $11.15 \%$ & $75.15 \%$ & $93.25 \%$ \\
\hline $.2-3$ & $12.95 \%$ & $80.10 \%$ & $94.15 \%$ & $12.20 \%$ & $80.45 \%$ & $95.40 \%$ & $10.85 \%$ & $77.00 \%$ & $92.85 \%$ \\
\hline $1.2-4$ & $11.75 \%$ & $78.20 \%$ & $94.90 \%$ & $12.20 \%$ & $79.35 \%$ & $95.30 \%$ & $7.70 \%$ & $75.40 \%$ & $92.30 \%$ \\
\hline
\end{tabular}

\footnotetext{
${ }^{4}$ Normal program execution takes less then ten seconds for all kernels.
} 


\subsection{Results}

Table 4 shows the reliability results for each version (rows) and each kernel $/ \lambda_{k}$ (columns). As expected, results improve with decreasing $\lambda_{k}$. At $\lambda_{k}=0.01,44.65-$ $84.4 \%$ of all runs using the original version complete with correct results. For the NMR versions, increasing $N$ does not improve reliability in our evaluations. This is due to the increased fault probability of the majority voter as $N$ increases. The number of clauses for $N=3,5$, and 7 is 3,10 , and 35 , respectively. The $1.2 \mathrm{n}$ versions and the $3 \mathrm{MR}$ version achieve similar reliabilities. With the exception of the Gaussian kernel, the versions produced by our approach achieve among the best reliabilities for all experiments. We discuss the results of the Gaussian kernel more in Section 5 .

Table 5 shows the performance results. An "-" entry indicates that no execution terminated with correct results. Performance stays somewhat constant over all $\lambda_{k}$ for all versions except OPT. This is expected for the original and NMR versions but surprising for the $1.2 \mathrm{n}$ versions. These results indicate that we tend to end up in the same stage independent of $\lambda_{k}$. The fact that performance cost decreases for OPT can be understood by looking at Table 2 the number of executions used decreases for decreasing $\lambda_{k}$. These numbers should not be used to assess general performance overhead of redundant execution. Our earlier work [15] shows that we need to hide memory latencies to keep the overhead low. This requires optimizations such as SIMDization and versioning to make use of parallel resources.

Figure 2 shows the geometric means of the reliability and performance results for all runs, excluding those of the Gaussian kernel. For each version, OPT achieves marginally better reliability than the other resilient versions. At the same time, if $\lambda_{k}$ is sufficiently low, its performance can be even better then 3MR (which indiscriminately adds redundancy in cases where our approach does not). For high $\lambda_{k}$ the execution overhead is quite high.
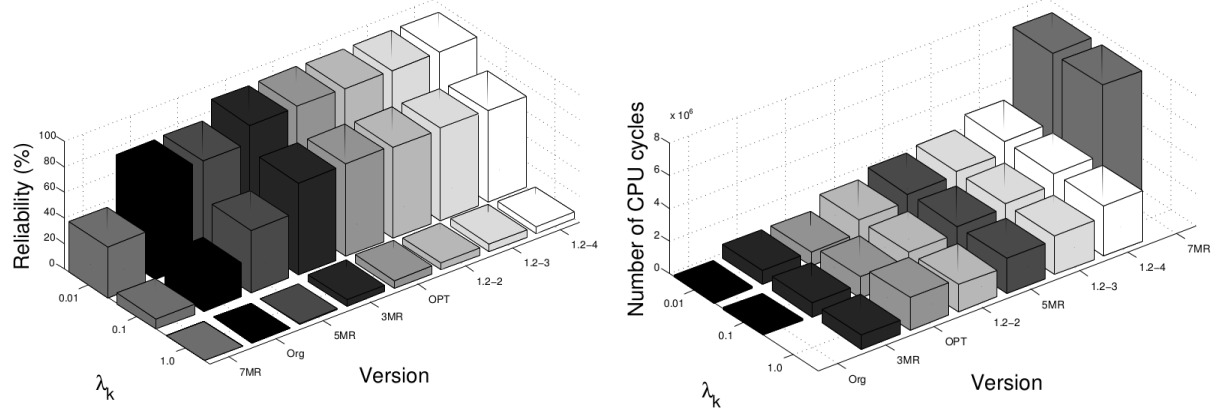

Fig. 2. Geometric Mean of Reliability (left) and Performance (right) Results 
Table 5. Median CPU Cycles for Each Kernel Version at Each $\lambda_{k}$

\begin{tabular}{|c|c|c|c|c|c|c|c|c|c|}
\hline \multirow[t]{3}{*}{ Version } & \multicolumn{9}{|c|}{ Kernel } \\
\hline & \multicolumn{3}{|c|}{ Approx-Log } & \multicolumn{3}{|c|}{ Determinant } & \multicolumn{3}{|c|}{ Gaussian } \\
\hline & $\lambda_{k}=1.0$ & $\lambda_{k}=0.1$ & $\lambda_{k}=0.01$ & $\lambda_{k}=1.0$ & $\lambda_{k}=0.1$ & $\lambda_{k}=0.01$ & $\lambda_{k}=1.0$ & $\lambda_{k}=0.1$ & $\lambda_{k}=0.01$ \\
\hline Orig & & 89380 & 89380 & 64198 & 63986 & 63986 & & 107258 & 107192 \\
\hline 3MR & 226534 & 225818 & 225738 & 107406 & 106966 & 106900 & 313871 & 308869 & 308337 \\
\hline 5MR & 479330 & 478074 & 477918 & 200758 & 200898 & 200832 & & 1913594 & 1912978 \\
\hline 7MR & & 2031302 & 2031017 & 421331 & 520667 & 520535 & & 6223762 & 6223072 \\
\hline OPT & 358369 & 219939 & 219859 & 419488 & 197007 & 101368 & & & \\
\hline $2-2$ & 399996 & 398956 & 98862 & 159122 & 158592 & 158496 & 1304283 & 1297262 & 1296546 \\
\hline $1.2-3$ & 576538 & 575591 & 575 & 207896 & 2077 & 207671 & 3158828 & 3153470 & 3152882 \\
\hline $1.2-4$ & 750603 & 750004 & 749956 & 255271 & 256290 & 256298 & 4197134 & 4194096 & 4193656 \\
\hline \multirow[t]{3}{*}{ Version } & \multicolumn{9}{|c|}{ Kernel } \\
\hline & \multicolumn{3}{|c|}{ Mincomp } & \multicolumn{3}{|c|}{ Mintime } & \multicolumn{3}{|c|}{ Bubblesort } \\
\hline & $\lambda_{k}=1.0$ & $\lambda_{k}=0.1$ & $\lambda_{k}=0.01$ & $\lambda_{k}=1.0$ & $\lambda_{k}=0.1$ & $\lambda_{k}=0.01$ & $\lambda_{k}=1.0$ & $\lambda_{k}=0.1$ & $\lambda_{k}=0.01$ \\
\hline Orig & & 124310 & 124310 & & 135194 & 135194 & & 178702 & 178702 \\
\hline 3MR & 2250657 & 2249985 & 2249901 & 2250653 & 2249989 & 2249901 & 3689882 & 3689220 & 3689136 \\
\hline 5MR & 6855928 & 6854958 & 6854864 & 6855988 & 6854958 & 6854866 & 9523724 & 9522746 & 9522652 \\
\hline 7MR & & 21741416 & 21741266 & & 30197146 & 30197008 & & 30197151 & 30197008 \\
\hline OPT & 6959278 & 4671986 & 2078021 & 8224106 & 5503566 & 2890332 & 3727170 & 3266087 & 3266005 \\
\hline $1.2-2$ & 5231408 & 5233664 & 5233950 & 5247188 & 5249624 & 5249954 & 7188489 & 7190031 & 7190215 \\
\hline $1.2-3$ & 7674798 & 7686217 & 7687520 & 7714317 & 7726648 & 7728076 & 10515264 & 10525612 & 10526772 \\
\hline $1.2-4$ & 10053976 & 10080261 & 10082966 & 10131521 & 10157786 & 10160536 & 13786320 & 13811295 & 13813971 \\
\hline
\end{tabular}

\section{Discussion}

We have introduced an algorithm to minimize the number of redundant executions for instructions in a CFG. This algorithm considers both the reliability of the instruction and its performance cost, and the surrounding framework represents a promising first cut at an automated solution. Nonetheless, we find that our current algorithm can be overly conservative in that it calculates the maximum number of redundant executions, which may, in turn, degrade reliability. For instance, in Section 4, the OPT version of the Gaussian kernel achieves a $0 \%$ reliability. This kernel makes heavy use of multiplication, which is our most unreliable operation due to the area of the multiplier.

Looking at Table 4, we see that Gaussian is not very resilient. The reliability at $\lambda_{k}=0.01$ is comparable to that of the determinant kernel at $\lambda_{k}=0.1$. Table 2 shows that our algorithm deduces that we need as many as 31 replications for most of the statements. The 1.mn voter that we introduced to cope with high $N$ does not have the desired effect in this case. Performing redundant execution with just NMR, on the other hand, is not a feasible solution. The overhead of majority voting as $N$ increases becomes very high (for both performance and reliability). This example highlights the need to consider not just the probability of correct execution but also the uncertainty that we associate with this probability. When the uncertainty becomes too high, we could then include a limited amount of replay (or other construction) to bring down the potential overhead.

We have not considered the case of optimizing performance/reliability over the entire acyclic CFG (but rather over each basic block individually). For this we would need to combine contributions from mutually exclusive control-flow paths. An affine join function could be used for this, but then we would need to include 
posynomial equality constraints, and geometric programming does not allow such constraints, in general. Allowing these makes it a signomial programming [9] problem, for which no efficient optimization algorithm is known to exist.

Although we show benefits over $3 \mathrm{MR}$, the improvements are marginal with our current approach. Nonetheless, the fact that our approach considers more variables makes it applicable to more complex environments (for instance, where only a subset of the instructions are faulty). We have not investigated such scenarios because the current study has highlighted the importance of considering how the uncertainty of our probabilistic flow calculations increases with the number of instructions in the section of the CFG we target. In particular, we have shown that schemes that only add redundant executions as needed can outperform NMR schemes.

\section{Conclusion}

One problem in using a redundant execution scheme is how to find the number of executions to use for each instruction. We show an algorithm for approximating this number while taking into account performance costs. Our evaluations show that it is possible to achieve good reliability while still minimizing performance overheads. We improve reliability by up to $58 \%$ compared to the original versions of our benchmarks. The median performance cost is up to $8 \%$ lower then triple modular redundancy.

Our results vary considerably with the assumed probability of an instruction's successful execution. If this probability is low, we conservatively recommend a high number of redundant versions. Adding more redundancy may be harmful, since it gives the faulty environment more changes to alter the semantics of the execution. Determining this probability is of course a big problem in itself. Although our 1.mn voter relieves some of these problems, it does not represent a general solution. Our results however show that reliability/performance improve if we only execute versions as needed to cope with this uncertainty. We believe a better optimization algorithm would factor this uncertainty into decisions.

Acknowledgments. The authors thank Alen Bardizbanyan and Kasyab Subramaniyan for helping to synthesize the MIPS processor.

\section{References}

1. Cappello, F., Geist, A., Gropp, B., Kale, L., Kramer, B., Snir, M.: Toward Exascale Resilience. International Journal of High Performance Computing Applications 23(4), 374-388 (2009)

2. Engelmann, C., Ong, H.H., Scott, S.L.: The Case for Modular Redundancy in Large-Scale High Performance Computing Systems. In: Proc. IASTED International Conference on Parallel and Distributed Computing and Networks (PDCN), pp. 189-194 (February 2009), Related work 
3. Li, D., Lee, S., Vetter, J.S.: Evaluating the viability of application-driven cooperative CPU/GPU fault detection. In: an Mey, D., et al. (eds.) Euro-Par 2013. LNCS, vol. 8374, pp. 670-679. Springer, Heidelberg (2014)

4. Fiala, D., Mueller, F., Engelmann, C., Riesen, R., Ferreira, K., Brightwell, R.: Detection and Correction of Silent Data Corruption for Large-scale High-performance Computing. In: Proceedings of the International Conference on High Performance Computing, Networking, Storage and Analysis, pp. 78:1-78:12 (2012)

5. Elliott, J., Kharbas, K., Fiala, D., Mueller, F., Ferreira, K., Engelmann, C.: Combining Partial Redundancy and Checkpointing for HPC. In: Proceedings of the International Conference on Distributed Computing Systems (ICDCS 2012), pp. 615-626 (2012)

6. Shamsunder, R., Rosenkrantz, D.J., Ravi, S.S.: Exploiting Data Flow Information in Algorithm-Based Fault Tolerance. In: Proc. International Symposium on FaultTolerant Computing (FTCS), pp. 280-289 (June 1993)

7. Lu, G., Zheng, Z., Chien, A.A.: When is Multi-version Checkpointing Needed? In: Proc. 3rd Workshop on Fault-Tolerance for HPC at Extreme Scale (FTXS), pp. 49-56 (June 2013)

8. Misailovic, S., Carbin, M., Achour, S., Zichao, Q., Rinard, M.: Reliability-Aware Optimization of Approximate Computational Kernels with Rely. MIT-CSAIL-TR2014-001 (January 2014)

9. Boyd, S., Kim, S.-J., Vandenberghe, L., Hassibi, A.: A tutorial on geometric programming. Optimization and Engineering 8(1), 67-127 (2007)

10. Carbin, M., Misailovic, S., Rinard, M.C.: Verifying Quantitative Reliability for Programs That Execute on Unreliable Hardware. In: Proc. SIGPLAN International Conference on Object Oriented Programming Systems Languages \& Applications (OOPSLA), pp. 33-52 (October 2013)

11. Quinlan, D., Liao, C.: The ROSE Source-to-Source Compiler Infrastructure. In: Cetus Users and Compiler Infrastructure Workshop, with the International Conference on Parallel Architectures and Compilation Techniques, PACT (October 2011)

12. Grant, M., Boyd, S.: CVX: Matlab Software for Disciplined Convex Programming, version 2.0 beta (June 2014), http://cvxr.com/cvx

13. Grant, M.C., Boyd, S.P.: Graph Implementations for Nonsmooth Convex Programs. In: Blondel, V., Boyd, S., Kimura, H. (eds.) Recent Advances in Learning and Control. LNCIS, vol. 371, pp. 95-110. Springer, Heidelberg (2008)

14. Binkert, N., Beckmann, B., Black, G., Reinhardt, S.K., Saidi, A., Basu, A., Hestness, J., Hower, D.R., Krishna, T., Sardashti, S., Sen, R., Sewell, K., Shoaib, M., Vaish, N., Hill, M.D., Wood, D.A.: The Gem5 Simulator. Computer Architecture News 39(2), 1-7 (2011)

15. Lidman, J., Quinlan, D.J., Liao, C., McKee, S.A.: ROSE:FTTransform - A Sourceto-Source Translation Framework for Exascale Fault-Tolerance Research. In: Proc. 2nd Workshop on Fault-Tolerance for HPC at Extreme Scale (FTXS), pp. 1-6 (June 2012)

16. Rinard, M., Cadar, C., Dumitran, D., Roy, D.M., Leu, T., Beebee Jr., W.S.: Enhancing Server Availability and Security Through Failure-Oblivious Computing. In: Proc. 6th Symposium on Operating Systems Design \& Implementation (OSDI), p. 21 (Decedmber 2004) 